\title{
APPLICATION OF THE EXTREMUM PRINCIPLE TO INVESTIGATING CERTAIN EXTREMAL PROBLEMS
}

BY

\author{
L. MIKOEAJCZYK AND S. WALCZAK
}

\begin{abstract}
Denote by $C, K, X$, respectively, a complex plane, the disc $\{z \in \mathbf{C}$ : $|z|<1\}$ and any compact Hausdorff space. Denote by $P$ a set of probabilistic measures defined on Borel subsets of the space $X$. For $\mu \in P$, let $f(z)=$ $\int_{X} q(z, t) d \mu, z \in K$, and $\mathscr{F}=\{f: \mu \in P\}$. Consider a finite sequence of real functions $F_{0}, F_{1}, \ldots, F_{m}$ defined in the space $R^{2 n}$. Let $\zeta_{1}, \ldots, \zeta_{k}$ be fixed points of the disc $K$ and $\eta(f)=\left[\right.$ re $f^{(0)}(\zeta)$, im $f^{(0)}\left(\zeta_{1}\right), \ldots$, re $f^{\left(n_{1}\right)}\left(\zeta_{1}\right)$, $\operatorname{im} f^{\left(n_{1}\right)}\left(\zeta_{1}\right) ; \ldots$, re $f^{(0)}\left(\zeta_{k}\right), \operatorname{im} f^{(0)}\left(\zeta_{k}\right), \ldots$, re $\left.f^{\left(n_{k}\right)}\left(\zeta_{k}\right), \operatorname{im} f^{\left(m_{k}\right)}\left(\zeta_{k}\right)\right]$, where $f \in \mathscr{F}$, $n=n_{1}+\cdots+n_{k}+k$. Let $F_{j}(f)=F_{j}(\eta(f)), j=0,1, \ldots, m$. We consider the following extremal problem. Determine a minimum of the functional $F_{0}(f)$ under the conditions $F_{j}(f)<0, j=1,2, \ldots, m, f \in \mathscr{F}$. We apply the extremum principle to solve this problem. In the linear case this problem was investigated in [11].
\end{abstract}

Introduction. In their monograph under the title "Theory of Extremal Problems" [1], A. D. Joffe and W. M. Tikhomirov gave a general method of investigating extremal problems in Banach spaces. In this method a theorem called the extremum principle is of essential importance. From the extremum principle one can derive, as it turns out, many theorems of the calculus of variations and those of the theory of control. In the present paper the extremum principle is applied to examining extremal problems in certain families of complex functions which have structural representation given by L. Brickman, T. H. MacGregor and D. R. Wiken (cf. [11]). On the basis of the extremum principle, there has been proved a general theorem characterizing extremal functions. From this theorem one can obtain, in particular, many results obtained earlier by other authors. In further considerations the terms family of functions, class of functions, set of functions, will be used as equivalent ones.

1. Introductory notes. By an extremal problem we mean, similarly as in monograph [1], a problem for a conditional extremum under various side conditions.

Extremal problems in various families of complex functions were investigated, among others, in papers [2]-[10]. Most problems examined were considered in those families of complex functions which have an integral representation. The considerations in the present paper will also concern these kinds of classes of complex functions.

Let us denote by $C, K, X$, respectively, a complex plane, the disc $\{z \in \mathbf{C}$ : $|z|<1\}$ and any compact Hausdorff space. We assume that the mapping $q$ : $K \times X \rightarrow C$ possesses the following properties. 
(i) For every $t \in X$ the mapping $z \rightarrow q(z, t)$ is analytic in $K$,

(ii) for every $z \in K$ the mapping $t \rightarrow q(z, t)$ is continuous on $X$,

(iii) for every $r, 0<r<1$, there exists a number $M_{r}>0$ such that $|q(z, t)|<M_{r}$ for $|z|<r$ and for $t \in X$.

Denote by $\mathscr{P}$ a set of probabilistic measures defined on Borel subsets of the sp:ace $X$. For $\mu \in \mathscr{P}$, let

$$
f_{\mu}(z)=\int_{X} q(z, t) d \mu(t), \quad z \in K,
$$

and let $\mathscr{F}=\left\{f_{\mu}: \mu \in \mathscr{P}\right\}$.

It is possible to show (cf. [11]) that

(a) every function of the set $\mathcal{F}$ is analytic in the disc $K$,

(b) the mapping $\mu \rightarrow f_{\mu}$ is continuous (under the weak-*-topology induced from the space $C(X)^{*}$ and under the topology of almost uniform convergence on $\mathscr{F}$ ),

(c) the set $\mathscr{F}$ is compact and it is a closed convex hull of the set of functions $\{z \rightarrow q(z, t): t \in X\}$,

(d) the functions $z \rightarrow q(z, t), t \in X$, are the only possible vertical points of the set $\mathscr{F}$. If $t_{0} \in X$ and the condition

$$
q\left(z, t_{0}\right)=\int_{X} q(z, t) d \mu(t), \quad z \in K,
$$

holds only for $\mu=\delta_{t_{0}}$, where $\delta_{t_{0}}$ denotes a Dirac measure at the point $t_{0}$, then the function $z \rightarrow q\left(z, t_{0}\right)$ is a vertical point of the set $\mathscr{F}$. In particular, if the mapping $\mu \rightarrow f_{\mu}$ is one-to-one, every function $z \rightarrow q(z, t), t \in X$, is some vertical point of the set $\mathscr{F}$.

As is known, one of the most familiar representations of form (1) is the Herglotz representation for analytic functions with real parts positive in the disc $K$. In this case the mapping $\mu \rightarrow f_{\mu}$ is one-to-one (cf. [12, p. 40]). Many interesting families of analytic functions possess a representation of form (1), where $X=[a, b], a<b$, while $\mathcal{P}=\mathscr{P}(a, b)$ is a set of measures cumulated on the segment $[a, b]$, such that

$$
\int_{a}^{b} d \mu(t)=1
$$

In further considerations, to shorten the notation, instead of (1) we shall write

$$
f(z)=\int_{X} q(z, t) d \mu(t) .
$$

On the basis of the properties of vertical points, in paper [11, §3] there have been proved some interesting theorems concerning linear complex functionals defined on chosen families of complex functions which have a representation of form (1). In the present paper a case of nonlinear functionals is being investigated.

2. Formulation of extremal problem. Let $m, n_{1}, n_{2}, \ldots, n_{k}$ be fixed natural numbers. Consider a finite sequence of real functions $F_{0}, F_{1}, \ldots, F_{m}$ defined in the space $R^{2 n}$, where $n=n_{1}+n_{2}+\cdots+n_{k}+k$. We assume that these functions are of the class $C^{(1)}$. 
Let $\zeta_{1}, \zeta_{2}, \ldots, \zeta_{k}$ be fixed distinct points of the disc $K$. Denote by $\eta$ a vector of the form

$$
\begin{aligned}
\eta(f)=\left[\operatorname{re} f^{(0)}\left(\zeta_{1}\right), \operatorname{im} f^{(0)}\left(\zeta_{1}\right), \ldots, \operatorname{re} f^{\left(n_{1}\right)}\left(\zeta_{1}\right), \operatorname{im} f^{\left(n_{1}\right)}\left(\zeta_{1}\right)\right. \\
\left.\ldots ; \operatorname{re} f^{(0)}\left(\zeta_{k}\right), \operatorname{im} f^{(0)}\left(\zeta_{k}\right), \ldots, \operatorname{re} f^{\left(n_{k}\right)}\left(\zeta_{k}\right), \operatorname{im} f^{\left(n_{k}\right)}\left(\zeta_{k}\right)\right],
\end{aligned}
$$

where $f \in \mathcal{F}, f^{(0)} \equiv f$.

We introduce the notation

$$
\text { re } f^{i}\left(\zeta_{j}\right)=u_{j}^{i}, \quad \operatorname{im} f^{(i)}\left(\zeta_{j}\right)=v_{j}^{i} \quad \text { for } i=0,1, \ldots, n_{j} \text { and } j=1,2, \ldots, k \text {. }
$$

With notation (5) vector (4) has the form

$$
\eta(f)=\left[u_{1}^{0}, v_{1}^{0}, \ldots, u_{1}^{n_{1}}, v_{1}^{n_{1}} ; \ldots ; u_{k}^{0}, v_{k}^{0}, \ldots, u_{k}^{n_{k}}, v_{k}^{n_{k}}\right] .
$$

We construct a finite sequence of real functionals

$$
F_{j}(f)=F_{j}(\eta(f)), \quad j=0,1, \ldots, m,
$$

defined on the family $\mathscr{F}$.

We shall consider the following extremal problem.

Problem 1. Determine a minimum (maximum) of the functional $F_{0}(f)$ under the conditions $F_{j}(f)<0, j=1,2, \ldots, m, f \in \mathscr{F}$.

REMARK 1. Equality constraints will not be paid respect to in general considerations, since every equality $F_{j}(f)=0$ can be represented as a conjunction of two inequalities $F_{j}(f)<0$ and $-F_{j}(f)<0$.

3. Extremum principle and theorem on extremal measures. Let $\varphi_{0}, \varphi_{1}, \ldots, \varphi_{m}$ be a finite sequence of real functionals defined on some nonempty and convex subset $U$ of the given space. Let us denote by $L$ a function of the form

$$
L\left(u, \lambda_{0}, \lambda_{1}, \ldots, \lambda_{m}\right)=\sum_{i=1}^{m} \lambda_{i} \varphi_{i}(u)
$$

where $u \in U$, while $\lambda_{0}, \lambda_{1}, \ldots, \lambda_{m}$ are given real numbers. Function (8) is called a Lagrange function, whereas the constants $\lambda_{i}, i=0,1, \ldots, m$ are Lagrange multipliers. In our further considerations we shall make use of the following theorem.

THEOREM 1 (EXTREMUM PRINCIPLE). If

I. $u_{0} \in U$ and $\varphi_{i}\left(u_{0}\right)<0, i=1,2, \ldots, m$,

II. $u_{0}$ is the argument of the minimum of the functional $\varphi_{0}$ on the set $U$ under the constraints $\varphi_{i}(u)<0, i=1,2, \ldots, m$,

III. the vector function $\varphi=\left[\varphi_{0}, \varphi_{1}, \ldots, \varphi_{m}\right]$ satisfies the following weak condition of convexity on the set $U$. For any $u_{1}, u_{2} \in U$ and $\alpha \in[0,1]$ there exists $u \in U$ such that

$$
\varphi_{i}(u)<\alpha \varphi_{i}\left(u_{1}\right)+(1-\alpha) \varphi_{i}\left(u_{2}\right) \text { for } i=0,1, \ldots, m,
$$

then there exist Lagrange multipliers $\lambda_{i}>0, i=0,1, \ldots, m$, not vanishing simultaneously such that

$$
\begin{aligned}
& \text { (a) } L\left(u_{0}, \lambda_{0}, \lambda_{1}, \ldots, \lambda_{m}\right)=\min _{u \in U} L\left(u, \lambda_{0}, \lambda_{1}, \ldots, \lambda_{m}\right) \text {, } \\
& \text { (b) } \lambda_{i} \varphi_{i}\left(u_{0}\right)=0 \text { for } i=1, \ldots, m \text {. }
\end{aligned}
$$


The above theorem follows directly from Theorem 3 formulated in monograph [1, pp. 79-80].

RemarK 2. If the functionals $\varphi_{i}, i=0,1, \ldots, m$, are convex, condition (9) is satisfied for $u=\alpha u_{1}+(1-\alpha) u_{2}$.

Let $v=v(t)$ be a continuous real function defined on the space $X$ mentioned in introductory notes.

Denote by $E$ the following set $E=\{\tau \in X: v(\tau)=M\}$, where $M=$ $\max _{t \in X} v(t)$.

We shall prove

THEOREM 2. If

$$
\int_{X} v(t) d \mu_{*}(t)=\max _{\mu \in \Phi} \int_{X} v(t) d \mu(t),
$$

then the measure $\mu_{*}$ is cumulated on the set $E$.

Proof. The space $X$ can be represented in the form $X=\cup_{k=1}^{\infty} X_{k} \cup E$, where

$$
\begin{gathered}
X_{k}=\left\{t \in X,\left(1-\frac{1}{k}\right)(M-m)+m<v(t)<\left(1-\frac{1}{k+1}\right)(M-m)+m\right\}, \\
M=\max _{t \in X} v(t), \quad m=\min _{t \in X} v(t) .
\end{gathered}
$$

The sets $X_{k}, k=1,2, \ldots$, and $E$ are pairwise disjoint, and thus $\mu_{*}(X)=$ $\sum_{k=1}^{\infty} \mu_{*}\left(X_{k}\right)+\mu_{*}(E)$. Suppose that the measure $\mu_{*}$ is not cumulated on the set $E$, i.e., $\mu_{*}(E)<\mu_{*}(X)=1$. And consequently, there exists some $k$ such that $\mu_{*}\left(X_{k}\right)=$ $a>0$.

Let $\bar{\mu} \in \mathcal{P}$ be any measure cumulated entirely on the set $E$. We have

$$
\begin{aligned}
\int_{X} v(t) d \mu_{*} & =\int_{X_{k}} v(t) d \mu_{*}+\int_{X \backslash X_{k}} v(t) d \mu_{*} \\
& \leqslant\left[\left(1-\frac{1}{k+1}\right)(M-m)+m\right] \mu_{*}\left(X_{k}\right)+M \mu_{*}\left(X \backslash X_{k}\right)<M .
\end{aligned}
$$

Since $\int_{X} v(t) d \bar{\mu}=M$, inequality (11) contradicts the assumption of Theorem 2 .

4. Application of the extremum principle. Basing ourselves on Theorems 1 and 2 , we shall prove a general necessary condition for the existence of the extremum in Problem 1.

The Lagrange function for Problem 1 has the form

$$
L\left(f, \lambda_{0}, \lambda_{1}, \ldots, \lambda_{m}\right)=\sum_{j=0}^{m} \lambda_{j} F_{j}(f) \text {. }
$$

Let $f_{*}, f_{*}(z)=\int_{X} q(z, t) d \mu_{*}(t)$ denote a solution of Problem 1. Such a solution does exist provided that the set

$$
W=\left\{f \in \mathscr{F}: F_{j}(f)<0, j=1,2, \ldots, m\right\}
$$

is not empty. 
Let $w$ be a function of the form

$$
w(t)=\sum_{s=1}^{k} \sum_{i=0}^{n_{s}}\left(A_{s}^{i} \operatorname{re~} q^{(i)}\left(\zeta_{s}, t\right)+B_{s}^{i} \operatorname{im} q^{(i)}\left(\zeta_{s}, t\right)\right),
$$

where $t \in X$,

$$
A_{s}^{i}=\left.\frac{\partial L\left(f, \lambda_{0}, \lambda_{1}, \ldots, \lambda_{m}\right)}{\partial u_{s}^{i}}\right|_{f=f_{*}} \quad B_{s}^{i}=\left.\frac{\partial L\left(f, \lambda_{0}, \lambda_{1}, \ldots, \lambda_{m}\right)}{\partial v_{s}^{i}}\right|_{f=f_{*}}
$$

and

$$
q^{(i)}\left(\zeta_{s}, t\right)=\left.\frac{\partial^{i}}{\partial \zeta^{i}} q(\zeta, t)\right|_{\zeta=\zeta_{s}}
$$

Denote by $E_{w}$ a set of the form $E_{w}=\left\{t \in X: w(t)=\max _{\tau \in X} w(\tau)\right\}$. The set is not empty since the function $w$ is continuous and the space $X$ compact.

We shall prove

THEOREM 3. If the function $f_{*}(z)=\int_{X} q(z, t) d \mu_{*}(t)$ is a solution of Problem 1 in which, for the function $F=\left[F_{0}, F_{1}, \ldots, F_{m}\right]$, the weak condition of convexity on the set $\mathscr{F}$ (condition III from Theorem 1) is satisfied, then the measure $\mu_{*}$ is cumulated on the set $E_{w}$.

Proof. From Theorem 1 we have

$$
L\left(f_{*}, \lambda_{0}, \lambda_{1}, \ldots, \lambda_{m}\right)=\min _{f \in \mathscr{F}} L\left(f, \lambda_{0}, \lambda_{1}, \ldots, \lambda_{m}\right),
$$

where $L$ is a function defined by formula (12).

Let $f$ be an arbitrary element of the family $\mathscr{F}$. Since $\mathscr{F}$ is a convex set, which follows directly from the definition of $\mathscr{F}$, then, together with the functions $f_{*}$ and $f$, to the set $\mathscr{F}$ there belongs a function

$$
f_{\varepsilon}=f_{*}+\varepsilon\left(f-f_{*}\right)
$$

with any $\varepsilon \in[0,1]$.

Taking account of (14) and (15), we get

$$
\left(\operatorname{grad} L\left(f_{*}, \lambda_{0}, \lambda_{1}, \ldots, \lambda_{m}\right), \eta\left(f-f_{*}\right)\right)+o(\varepsilon) / \varepsilon>0,
$$

where $o(\varepsilon) / \varepsilon \rightarrow 0$ with $\varepsilon \rightarrow 0$.

After simple transformations we obtain

$$
\sum_{s=1}^{k} \sum_{i=0}^{n_{s}}\left(A_{s}^{i} \operatorname{re} f^{(i)}\left(\zeta_{s}\right)+B_{s}^{i} \operatorname{im} f^{(i)}\left(\zeta_{s}\right)\right)<\sum_{s=1}^{s} \sum_{i=0}^{n_{s}}\left(A_{s}^{i} \operatorname{re} f_{*}^{(i)}\left(\zeta_{s}\right)+B_{s}^{i} \operatorname{im} f_{*}^{(i)}\left(\zeta_{s}\right)\right)
$$

Hence, taking account of (3) and (13), we get

$$
\int_{X} w(t) d \mu(t)<\int_{X} w(t) d \mu_{*}(t)
$$

where $\mu, \mu_{*} \in \mathscr{P}$.

Making use of Theorem 2, we obtain the proposition of the theorem being proved. 
Corollary 1. If

$1^{\circ} X \subset R$,

$2^{\circ} w(t) \neq$ const.,

$3^{\circ}$ the mapping $X \ni t \rightarrow q(z, t)$ is analytic on the set $X$, then the extremal function $f_{*}$ is of the form

$$
f_{*}(z)=\sum_{i=1}^{N} \alpha_{i} q\left(z, t_{i}\right)
$$

where $N$ is some natural number, $\alpha_{i} \geqslant 0, i=1, \ldots, N, \sum_{i=1}^{N} \alpha_{i}=1, t_{i} \in X, i=$ $1, \ldots, N$.

Proof. It follows from the assumption that the function $w$ is analytic and is not identically constant. Consequently, the set $E_{w}$ is finite. The number of its elements is denoted by $N$. From equality (3) we obtain formula (16), which concludes the proof.

RemarK 3. Let $X \subset R$ and let $X$ be a compact set. The mapping $X \ni t \rightarrow a(t)$ is called analytic on this set if, for every point $t_{0} \in X$, there exists a neighbourhood $\left(t_{0}-\delta, t_{0}+\delta\right)$ in which $a(t)$ can be represented in the form

$$
a(t)=\sum_{n=0}^{\infty} a_{n}\left(t_{0}\right)\left(t-t_{0}\right)^{n} \quad \text { for } t \in\left(t_{0}-\delta, t_{0}+\delta\right) \cap X .
$$

5. On the possibility of certain generalizations of Problem 1. Let $\mathscr{D}$ be a compact subset of the space $R^{m}$, whereas $F_{0}, F_{1}, \ldots, F_{m}$ is a finite sequence of real functionals which are defined on the family $\mathcal{F}$ and have the form (7).

Consider the following

Problem 2. Determine a minimum of the functional $F_{0}(f)$ defined on the family $\mathscr{F}$ under the conditions

$$
\left(F_{1}(f), \ldots, F_{m}(f)\right) \in \mathscr{D}, \quad f \in \mathscr{F} .
$$

Let $f=f_{*}$ be a solution of Problem 2 .

If the set of functions $f \in \mathscr{F}$ which satisfy condition (17) is not empty, then, on account of the compactness of the set $\mathscr{D}$, the solution $f_{*}$ is sure to exist.

Put $F_{j}\left(f_{*}\right)=a_{j}, j=1,2, \ldots, m$. It is readily seen that the function $f=f_{*}$ will be the argument of the minimum of the functional $F_{0}$ under the conditions

$$
F_{j}(f)-a_{j}<0, \quad-F_{j}(f)+a_{j}<0, \quad j=1,2, \ldots, m .
$$

Hence we infer that the method of examining Problem 1 can also be adapted to examining Problem 2.

Let us still consider a case of complex functionals. Then let $F_{0}, F_{1}, \ldots, F_{m}$ be a finite sequence of continuous complex functionals of the form $F_{j}(f)=F_{j}(\eta(f))$, $j=0,1, \ldots, m$, defined on the family $\mathscr{F}$.

In this case the extremal problem will be formulated as follows.

Problem 3. Determine the set of values of the functional $F_{0}$ under the conditions

$$
F_{j}(f)=0, \quad j=1,2, \ldots, m .
$$

Denote by $\Delta_{0}$ the set of values of the functional $F_{0}$ under conditions (18) and by $\Gamma$ its boundary. 
One can show that the set $\Delta_{0}$ is compact. The function $f_{*}$ is called a boundary function with respect to the functional $F_{0}$ if $F_{0}\left(f_{*}\right) \in \Gamma$ and $F_{j}\left(f_{*}\right)=0, j=1$, $2, \ldots, m$.

Let us first consider a case when the set $\Delta_{0}$ is a convex set.

Note that in this case, if $f_{*}$ is a boundary function, there exists a number $a_{0} \in R$ such that $f_{*}$ is the argument of the extremum of the functional re $F_{0}$ under the conditions

$$
\begin{aligned}
\operatorname{im} F_{0}(f)-a_{0}=0, \quad \text { re } F_{j}(f)=0, \quad \operatorname{im} F_{j}(f) & =0, \\
j & =1,2, \ldots, m,
\end{aligned}
$$

or $f_{*}$ is the argument of the extremum of the functional im $F_{0}$ under the conditions

$$
\begin{aligned}
& \text { re } F_{0}(f)-a_{0}=0, \quad \text { re } F_{j}(f)=0, \quad \operatorname{im} F_{j}(f)=0 \text {, } \\
& j=1,2, \ldots, m \text {. }
\end{aligned}
$$

Hence it can be seen that Theorem 3 and Corollary 1 may also be applied to investigating Problem 3 in the case considered. With that end in view, one should replace equalities (19) and (20) by an equivalent system of inequalities and check whether the condition of weak convexity is satisfied.

Let us in turn consider a case when the set $\Delta_{0}$ is an arbitrary compact set.

The point $w_{0} \in \Gamma$ will be called a regular boundary point if there exists a point $\zeta \bar{\epsilon} \Delta_{0}$ such that $\left|w_{0}-\zeta\right|=\min _{w \in \Delta_{0}}|w-\zeta|$. The set of regular points is denoted by $\Gamma^{\prime}$. It is to be proved (cf. [13]) that the set $\Gamma^{\prime}$ is dense in $\Gamma$.

The function $f_{*} \in \mathscr{F}$ is called a boundary function regular with respect to the functional $F_{0}$ under conditions (18) if $F_{0}\left(f_{*}\right) \in \Gamma^{\prime}$.

It is not difficult to see that, if $f_{*}$ is a boundary function regular with respect to the functional $F_{0}$ under conditions (18), then it is the argument of the minimum of the real functional $\tilde{F}_{0}=\left|F_{0}-\zeta\right|^{2}$ for some $\zeta \in \Delta_{0}$.

So, if in the considerations we have carried out so far the functional $F_{0}$ is replaced by the functional $\tilde{F}_{0}$, then Theorem 3 and Corollary 1 may also be applied to investigating the set of values of complex functionals.

The above considerations concerning complex functionals will be illustrated by the following

EXAMPLE. Determine the set of values $\Delta_{0}$ of the functional $F_{0}(f)=f\left(\zeta_{1}\right)$ in the family of typically-real functions with fixed second coefficient $a_{2}=f^{\prime \prime}(0) / 2=c$, where $c \in[-2,2]$.

It is known (cf. [14]) that the family of typically-real functions coincides with the family $\mathcal{F}$ with $q(z, t)=z /\left(1-2 z \cos t+z^{2}\right)$ and $X=[-\pi, \pi]$. It is easy to see that the set $\Delta_{0}$ will be convex in this case. As we found before, every boundary function is at the same time an extremal function of the functional

$$
\text { re } F_{0}(f)=\left(f\left(\zeta_{1}\right)+\overline{f\left(\zeta_{1}\right)}\right) / 2
$$

under the conditions

$$
\begin{gathered}
\operatorname{im} F_{0}(f)=\left(f\left(\zeta_{1}\right)-\overline{f\left(\zeta_{1}\right)}\right) / 2 i-a_{0}=0, \\
\left(f^{\prime \prime}(0)+\overline{f^{\prime \prime}(0)}\right) / 2-c=0,
\end{gathered}
$$


or it is an extremal function of the functional

$$
\operatorname{im} F_{0}(f)=\left(f\left(\zeta_{1}\right)-\overline{f\left(\zeta_{1}\right)}\right) / 2 i
$$

under the conditions

$$
\begin{gathered}
\text { re } F_{0}(f)-a_{0}=\left(f\left(\zeta_{1}\right)+\overline{f\left(\zeta_{1}\right)}\right) / 2-a_{0}=0, \\
\left(f^{\prime \prime}(0)+\overline{f^{\prime \prime}(0)}\right) / 2-c=0,
\end{gathered}
$$

where $a_{0}$ is some real number.

It can easily be verified that in the case $\zeta_{1}=\bar{\zeta}_{1}$ the set $E_{w}$ defined in $\$ 4$ of the present paper contains at most three points, whereas in the case $\zeta_{1} \neq \bar{\zeta}_{1}$ at most four points. Consequently, the boundary function is of the form

$$
f_{*}(z)=\sum_{k=1}^{4} \frac{z}{1-2 z \cos t_{k}+z^{2}} \rho_{k},
$$

where $t_{k} \in[-\pi, \pi], \rho_{k} \geqslant 0, k=1,2,3,4, \Sigma_{k=1}^{4} \rho_{k}=1$. Since in this case the function $w$ (see [13]) is even, the points $t_{k}, k=1,2,3,4$, are symmetrically situated with respect to zero. And consequently, every boundary function can be represented in the form

$$
f_{*}(z)=\lambda \frac{z}{1-2 z \cos \tau_{1}+z^{2}}+(1-\lambda) \frac{z}{1-2 z \cos \tau_{2}+z^{2}},
$$

where $\lambda \in[0,1], \tau_{i} \in[0, \pi], i=1,2$.

The above result was obtained in some other way in paper [15].

REMARK 4. Most extremal problems considered in the theory of complex functions have the form: determine an extremum of the functional $F_{0}=F_{0}(f)$ defined in a given family of functions. If $F_{0}$ is a continuous functional, while the given family is connected, then the functional $F_{0}$ possesses the Darboux property and consequently, it satisfies the condition of weak convexity. By choosing the function $q(z, t)$ in a suitable way and applying Theorem 3 , one can obtain with no difficulty many familiar results for the families of Carathéodory, typically-real, starlike and other functions.

Literature in this field is very comprehensive and by and large well known. Many results and titles of reference books concerning this question can be found in [12] and [16]-[18] according to the references of the present paper.

\section{REFERENCES}

1. A. D. Joffe and W. M. Tikhomirov, Theory of extremal problems, "Nauka", Moscow, 1974. (Russian)

2. R. Robinson, Bounded univalent functions, Trans Amer. Math. Soc. 52 (1942), 426-449.

3. O. S. Nosenko, On domains of values of Stieltjes' functionals with equality constraints, Dopovidi Akad. Nauk Ukrain. RSR 1963. (Russian)

4. S. A. Kasaniuk, Mathematical programming and problems for a conditional extremum in special classes of analytic functions, Sibirsk. Mat. Z. 10 (1969).

5. L. Mikołajczyk, $A$ theorem on distortion for univalent p-symmetrical functions bounded in the circle $|z|<1$, Comment. Math. Prace Mat. 12 (1968).

6. I. A. Alexandrov and W. I. Popov, Optimal control and univalent functions, Ann. Univ. Mariae Curie-Skłodowska Sect. A 22 (1968/70).

7. G. S. Goodman, Univalent functions and optimal control, Ph.D. Thesis, Stanford Univ., 1968. 
8. S. Walczak, Method of examining conditional extrema in some families of complex functions, Bull. Acad. Polon. Sci. Sér. Sci. Math. Astronom. Phys. 24 (1976).

9. W. Tutschke, Über die Untersuchung von Problem der optimalen Steuerung in der Ebene mittels komplexer Methoden, Math. Nachr. 60 (1974).

10. S. Friedland and M. Schiffer, On coefficient regions of univalent functions, J. Analyse Math. 31 (1977).

11. L. Brickman, T. H. MacGregor and D. R. Wiken, Convex hulls of some classical families of univalent functions, Trans. Amer. Math. Soc. 156 (1971), 91-107.

12. Ch. Pommerenke, Univalent functions, Göttingen, 1975.

13. A. C. Schaeffer and D. C. Spencer, Coefficient regions for schlicht functions, Amer. Math. Soc. Colloq. Publ., no. 35, Amer. Math. Soc., Providence, R. I., 1950.

14. W. Rogoziński, Über positive harmonische Entwicklungen und typische-reelle Potenzreihen, Math. Z. 35 (1932).

15. A. Bielecki, J. Krzyż and Z. Lewandowski, On typically-real functions with preassigned second coefficient, Bull. Acad. Polon. Sci. Sér. Sci. Math. Astronom. Phys. 10 (1962), 205-208 .

16. G. M. Golusin, Geometric theory of functions of a complex variable, "Nauka", Moscow, 1966; English transl., Transl. Math. Monographs, vol. 26, Amer. Math. Soc., Providence, R. I., 1969.

17. G. Schober, Univalent functions. Selected topics, Lecture Notes in Math., vol 478, Springer-Verlag, Berlin and New York, 1975.

18. I. A. Alexandrov, Parametric continuations in the theory of univalent functions, Moscow, 1976. (Russian)

Institute of Mathematics, University of Kódź, ul. S. Banacha 22, 90-238 Kódź, Poland 